\title{
ESPACIO LIBRE INTEROCLUSAL EN LAS MORDIDAS PROFUNDAS: POSIBILIDAD DE EXTRUSIÓN DE LOS SECTORES POSTERIORES
}

\section{FREE SPACE IN DEEP OVERBITE WITH DENTAL ARCH: THE POSSIBILITY OF EXTRUSION}

\author{
Obando-Romero José-Alonzo ${ }^{1 *}$ \\ ${ }^{1}$ Esp. Ortodoncia y Ortopedia Maxilar, Docente 2da Esp. Ortodoncia y Ortopedia Maxilar Universidad Católica de Santa \\ María. Docente 2da Esp. Ortodoncia y Ortopedia Maxilar Universidad Andina del Cusco. Perú \\ jobando@ucsm.edu.pe
}

\begin{abstract}
Resumen
Los problemas verticales en las desarmonías dentomaxilares son muy comunes, la mordida profunda como característica de la clase II 2da división podría resolverse comprendiendo el problema en otra perspectiva, inmiscuyendo en el tratamiento otro tipo de opciones que la mera intrusión de los sectores anteriores. La participación de dispositivos como placas de levante posterior ayudan a modificar el plano oclusal, tratando las curvas de la oclusión, modificando el engrama muscular del paciente. Se realiza una revisión de la literatura sobre las posibles causas de la mordida profunda y la influencia de las placas de levante lateral en el Espacio libre interoclusal como característica individual especifica de la clase II 2da división. El uso de las placas de levante lateral traslada principios de la ortopedia funcional de los maxilares a la ortodoncia, modificación del plano oclusal, propiciando la erupción pasiva de sectores posteriores, creando una nueva relación sagital en el sector anterior.
\end{abstract}

Palabras clave: Mordida profunda, maloclusión, ortodoncia MT..

\begin{abstract}
Vertical problems in dentomaxillary disharmonies are very common, the deep bite characteristic of Class II 2nd division could be solved by understanding the problem from another perspective, interfering in the treatment with other options than the mere intrusion of previous sectors. The participation of devices like posterior lift plates help to modify the occlusal plane, treating the occlusion curves, modifying the patient's muscular engram. A literature review of the possible factors producing deep bite malocclusion and the influence of lateral elevator plates in the free interocclusal space as a specific individual characteristic of the class II 2 nd division was made. The use of lateral lift plates translates the functional orthopedic principles of the jaws into orthodontics, modifying the occlusal plane, favoring the passive eruption of posterior sectors and creating a new sagittal relationship in the anterior dental arch segment.
\end{abstract}

Key words: Ddeep bite, maloclusión, orthodontic.

\section{INTRODUCCIÓN}

Uno de los desequilibrios más comunes en la relación vertical de los maxilares es la mordida profunda, teniendo como normalidad un rango de 37,9 y $40 \%{ }^{1}$ y como hemos estudiado, las característica de su etiología es de orden multifactorial, de manera que si se realiza un listado de sus causas podemos encontrar factores genéticos, características étnicas, patrones musculares, hábitos, etc. ${ }^{2}$ Enfocándonos más puntualmente a desórdenes del desarrollo de la oclusión como factor etiológico, observando que sólo se podrá establecer el trípode oclusal, oclusión de molares permanentes y relación interincisiva, cuando no existan alteraciones sagitales, tal es, que exista una clase I dentaria. Los fenómenos de erupción activa, en primer lugar, y pasiva al finalizar la emergencia coronaria, permitirán que se desarrolle un plano oclusal más o menos recto. Es decir, una curva de Spee plana. Típico de la clase II dentaria, se rompe este equilibrio en el sector anterior y se genera una anomalía en la conformación del plano oclusal anterior. Con independencia de dónde esté asentada la alteración sagital de clase II, si en el maxilar superior, en la mandíbula o en ambos, la relación intermaxilar a nivel de los incisivos se verá severamente alterada. Los 


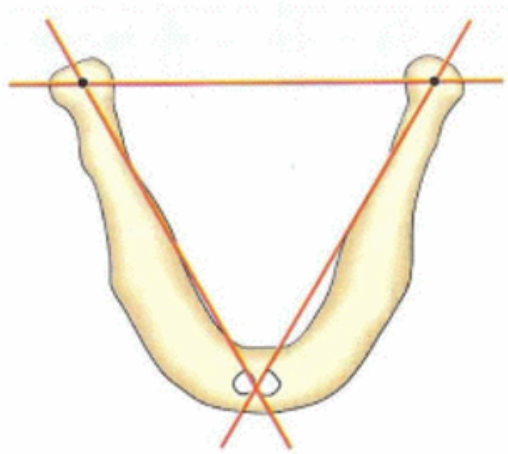

Fig. 1. Trípode oclusal, Con la erupción de los primeros incisivos se produce un cambio en las estructuras y se obtiene una centricidad mandibular Fuente: Barbara Fuentes, Unidad cráneo cervico mandibular, Universidad del Desarrollo, 2013

incisivos inferiores no encontrarán el apoyo anatómico de la cara palatina del incisivo superior y se romperá entonces el trípode oclusal.

La ausencia de contacto intermaxilar en el grupo dentario (incisivos y secundariamente caninos) por la mala relación sagital de los maxilares en la clase II, permite una sobre erupción de los incisivos inferiores y una manifestación de crecimiento vertical del reborde alveolar a modo de compensación de esta falta de oclusión, en virtud de los fenómenos de erupción activa y erupción pasiva. Esta sobre erupción será de mayor magnitud en función de la severidad de la clase II existente. ${ }^{2}$ De esta forma se genera una curva de Spee acentuada o profunda anterior que va en aumento durante la fase de crecimiento del paciente y puede continuar incluso una vez finalizado éste. Entonces en aquellos casos en que los incisivos superiores, ya sea por problemas de torque o de severas alteraciones sagitales, no encuentren el contacto con el labio inferior, pueden sufrir la sobre erupción por el crecimiento del reborde alveolar superior. ${ }^{3}$

En la resolución de las mordidas profundas se ha mencionado las placas de levante lateral para la modificación del plano oclusal por erupción pasiva de los sectores posteriores y así mejorar la relación vertical dentó-alveolar. Obviamente que este procedimiento tiene algunas indicaciones y contraindicaciones mencionadas con antelación en la bibliografía como biotipo, origen, edad, AFAI, estadio de maduración, cuantía de la displasia, etc. Estas placas son instaladas en las arcadas y tienen una injerencia muy importante en el complejo dentó-alveolar y repercuten en la altura facial antero inferior del paciente y en el ELI del paciente. Los objetivos seran:

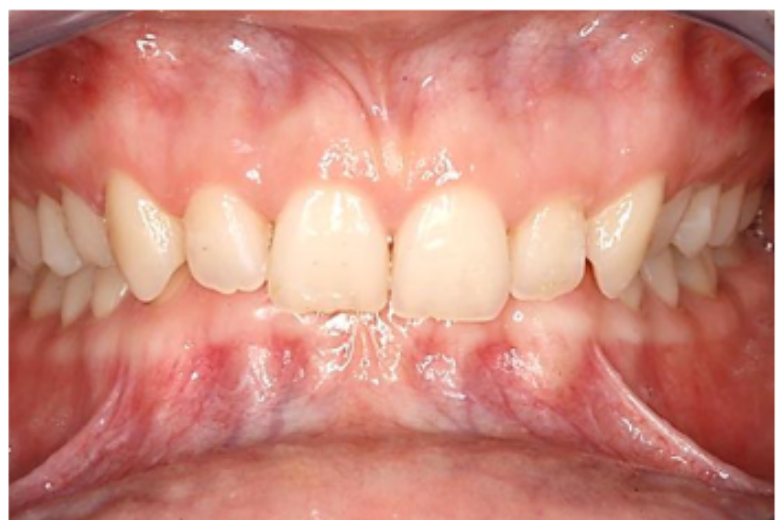

Fig. 2. Mordida Profunda característica propia de la clase II 2da división, curca de Spee muy acentuada. Apreciamos un over bite aproximadamente de $-10 \%$. Fuente: Propia

1) Tratar de esclarecer el posible manejo de la mordida profunda con respecto a su etiología

2) Ilustrar otro potencial manejo clínico de los problemas verticales.

3) Integrar y unificar criterios diagnósticos y complementarios de la rehabilitación oral, la ortodoncia y la ortopedia maxilar.

\subsection{ESPACIO LIBRE INTEROCLUSAL (ELI) EN LA CLA- SE II SEGUNDA DIVISIÓN}

El espacio libre interoclusal representa el espacio entre las superficies oclusales de las arcadas maxilares con la mandíbula en posición de reposo. Es el resultado de la diferencia entre la dimensión vertical postural o de reposo (DVP) y la dimensión vertical de oclusión (DVO). ${ }^{4,5} \mathrm{Se}$ considera en promedio de 2 a $4 \mathrm{~mm}$. Aunque algunos autores mencionan que varía de $2-3 \mathrm{~mm}$, mientras que otros dicen que varía de $1-3 \mathrm{~mm} .{ }^{6}$ Independientemente a la posición de la cabeza, que como ya sabemos existen muchas variantes de la misma durante el reposo y la vigilia. ${ }^{7}$ En la Clase II segunda división, una de sus características primordiales es la mordida profunda. ${ }^{8,9}$ Esta clase II presenta unos 7 a $9 \mathrm{~mm}$ de ELI.

Este fenómeno se explica por la hipertonicidad de los músculos masticatorios y la tendencia a la rotación anterior de la mandíbula condicionan una infraoclusión de los dientes posteriores que explica el aumento del espacio libre interoclusal cuando la mandíbula está en posición de reposo. $\mathrm{El}$ acortamiento de la distancia vertical entre ambas bases maxilares, por la presión muscular, lleva a intruir los molares y aumenta la sobremordida y el espacio libre..$^{3-10}$

\subsection{MODIFICACIÓN DEL PLANO OCLUSAL}

Se ha mencionado que para la resolución de mordidas profunda podríamos intruir incisivos, ${ }^{11,12}$ en pacientes con perfil cóncavo, patrón esquelético hipodivergente y altura 


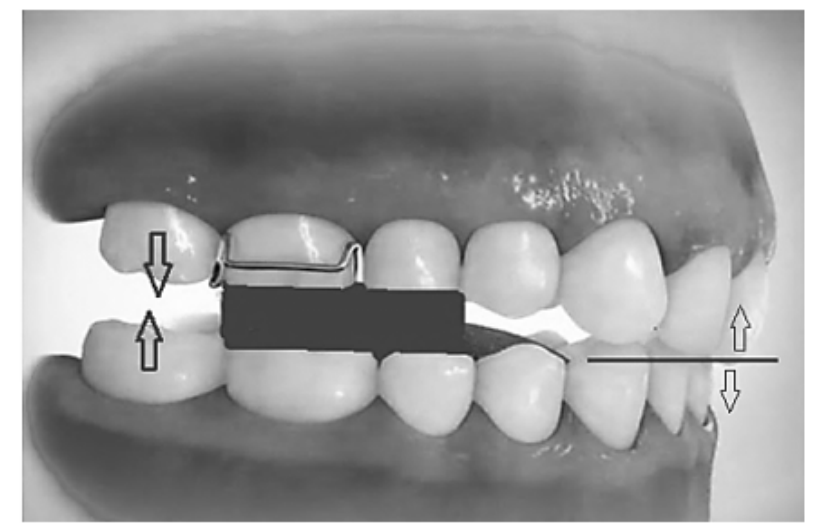

Fig. 3. Placa de levante lateral, modificación del plano oclusal propiciando la erupción pasiva de sectores posteriores, creando una nueva relación sagital en el sector anterior, propiciando y transfiriendo conceptos de la ortopedia funcional de los maxilares a la ortodoncia. Fuente: Propia

facial anteroinferior reducida, se indican los movimientos extrusivos de dientes posteriores para corregir la mordida profunda, los cuales deben hacerse paulatinamente para propiciar la adecuada adaptación neuromuscular y disminuir el riesgo de recidiva. ${ }^{13}$

Lo anterior se puede lograr con arcos para extrusión o elásticos pero éstos entregan fuerzas mayores no fisiológicas que repercuten sobre la estabilidad y el movimiento seguro de las molares. Por lo expuesto en el presente texto la instalación y uso de placas de levantamiento lateral (acrílicas de preferencia) podrían tener buenos resultados a la hora de tratar de normalizar las curvas de la Oclusión (Spee y Wilson) y conseguir un plano oclusal más "Plano". Su instalación y modo de acción sugiere una excitación neural dental proveniente del frote mismo de la mordida. Así en la figura 3 vemos como la modificación del plano oclusal con levantamientos laterales equidistantes en la clase II subdivisión 2 da con la respectiva evaluación de la masa dentaria (exodoncias de primeras bicúspides) o en combinación con mecánicas intrusivas (arcos base, minicrotornillos) ayudaría a restablecer el equilibrio oclusal y mejorar el ELI, llevando al caso a la estabilidad oclusal ideal.

\section{CONCLUSIONES}

-El tratamiento de las mordidas profundas puede ser visto de un enfoque más panorámico, teniendo como pilar fundamental el conocimiento profundo del desarrollo de sus posibles etiologías.

-El manejo de las implicancias de las curvas oclusales y el plano oclusal podrían tener nuevas perspectivas en el manejo de las mordidas profundas.
- El uso de las placas de levante lateral traslada principios de la ortopedia funcional de los maxilares a la ortodoncia, modificación del plano oclusal, propiciando la erupción pasiva de sectores posteriores, creando una nueva relación sagital en el sector anterior

Conflicto de intereses y financiamiento Los autores declaran no tener conflicto de intereses, haber cumplido con los requisitos de autoría y haber autofinanciado este artículo.

\section{Referencias}

1 Burstone CJ. Deep overbite correction by intrusion. Am J Orthod 1977; 72(1): 1-22.

2 Escobar P. Horacio, La curva de Spee, etiología y prevención en ortodoncia. Centro de estudios de ortodoncia, Gaceta Dental 2013; 109(12): 251-260.

3 Anibal, Alonso. Oclusión y Diagnóstico en Rehabilitación Oral. Ed. Panamericana, P. 372; 1999.

4 Uribe Restrepo, Gonzalo. Ortodoncia Teoría y Clínica. 2da edición, P. 232; 2010.

5 Beatriz Magali Cruz Moreno, Camen Elena Muñoz Gaviria, tratamiento ortodóncico de mordidas profundas. Rev Fac Odontol Univ Antioq 2010; 23(1): 158-173.

6 Ocampo, A.; Milena, Z.: Diagnóstico de las alteraciones verticales dentofaciales. Rev Fac Odont Univ de Antioquia 2009; Vol. 17(1): 121.128.

7 Chandrasekharan N. Revisión de las dentaduras completas. J Indian Prosthodont Soc. Jun 2013; 13(2): 68-70

8 Fonollosa, Josep. Diseño de Prótesis y Aparatos de Ortodoncia. Pág. 46

9 Proffit W.; Fields, Jr.; Sarver D. Ortodoncia Contemporánea. Ediciones Elsevier, Cuarta edición, 2008 p. 534-548; 287299 ;.

10 Kim J.; Akimoto S.; Shinji H.; Sato S.: Importance of vertical dimension and cant of occlusal plane in craniofacial development 2009. Int J Stomat \& Occlusion Med. Vol 2 (3), pp. 114-121.

11 Nanda R.; Kapila S. Terapias actuales en Ortodoncia. Ediciones Amolca. 2011 P. 186-198;

12 Nanda R. The differential diagnosis and treatment of excessive overbite. Dent Clin North Am 1981; 25(1): 69-84.

13 Nanda R. Correction of deep overbite in adults. Dent Clin North Am 1999; 41(1): 67-87.

14 Lagos A, Juárez I, Iglesias M. Necesidad de tratamiento periodontal e higiene oral en adolescentes de 12 años de Llanquihue. Scielo.2014;7(2).

Recibido: 05 de Junio de 2016

Aceptado: 15 de Junio de 2016 
